\title{
Robot-assisted radiofrequency ablation of primary and secondary liver tumours: early experience
}

\author{
Basri Johan Jeet Abdullah • Chai Hong Yeong • \\ Khean Lee Goh • Boon Koon Yoong • Gwo Fuang Ho • \\ Carolyn Chue Wai Yim • Anjali Kulkarni
}

Received: 22 May 2013 / Revised: 1 July 2013 / Accepted: 10 July 2013 / Published online: 9 August 2013

(C) The Author(s) 2013. This article is published with open access at Springerlink.com

\begin{abstract}
Objective Computed tomography (CT)-compatible robots, both commercial and research-based, have been developed with the intention of increasing the accuracy of needle placement and potentially improving the outcomes of therapies in addition to reducing clinical staff and patient exposure to radiation during CT fluoroscopy. In the case of highly inaccessible lesions that require multiple plane angulations, robotically assisted needles may improve biopsy access and targeted drug delivery therapy by avoidance of the straight line path of normal linear needles.

Methods We report our preliminary experience of performing radiofrequency ablation of the liver using a robotic-assisted CT guidance system on 11 patients (17 lesions).
\end{abstract}

\footnotetext{
B. J. J. Abdullah $(\square)$

Department of Biomedical Imaging, Faculty of Medicine, University of Malaya, 50603 Kuala Lumpur, Malaysia

e-mail: basrij@ummc.edu.my

C. H. Yeong

University of Malaya Research Imaging Centre, Faculty of Medicine, University of Malaya, Kuala Lumpur, Malaysia
}

\section{K. L. Goh}

Department of Internal Medicine, Faculty of Medicine, University of Malaya, 50603 Kuala Lumpur, Malaysia

B. K. Yoong

Department of Surgery, Faculty of Medicine, University of Malaya, 50603 Kuala Lumpur, Malaysia

\section{G. F. Ho}

Department of Oncology, Faculty of Medicine, University of Malaya, 50603 Kuala Lumpur, Malaysia

C. C. W. Yim

Department of Anesthesia, Faculty of Medicine, University of Malaya, 50603 Kuala Lumpur, Malaysia

\author{
A. Kulkarni \\ Perfint Healthcare Corporation, Florence, OR 97439, USA
}

Results/Conclusion Robotic-assisted planning and needle placement appears to have high accuracy, is technically easier than the non-robotic-assisted procedure, and involves a significantly lower radiation dose to both patient and support staff.

Key Points

- An early experience of robotic-assisted radiofrequency ablation is reported

- Robotic-assisted RFA improves accuracy of hepatic lesion targeting

- Robotic-assisted RFA makes the procedure technically easier with significant lower radiation dose

Keywords Robot - Radiofrequency ablation - Liver tumour . CT-guided $\cdot$ Interventional radiology

\section{Introduction}

Computed tomography (CT)-compatible robots have been developed and may soon be integrated into CT-guided renal mass ablation, hopefully reducing the radiation exposure to clinical staff and patients during CT fluoroscopy [1]. One recent study compared a preoperative computer-assisted optical needle tracking navigation system (KOELIS ${ }^{\circledR}$, Medtech Inc, Grenoble, France) with a CT-mounted robotic needle driver system $\left(\mathrm{AcuBot}^{\circledR}{ }^{\circledR}\right.$, Johns Hopkins University, Baltimore, MD, USA) and found improved accuracy (mean target distance 1.2 versus $5.8 \mathrm{~mm}, P<0.0001)$ and reduced targeting time (37 versus $108 \mathrm{~s}, P<0.0001)$ for the CT-mounted robotic needle driver system [2]. The authors demonstrated the potential of robotic needle guidance to improve needle interventions, demonstrating superiority over a commercial navigation system.

Even newer robotic-based image-guided procedures are in development including specialised robotically controlled "steerable" needles that may allow for access to previously 
inaccessible anatomical structures for improved biopsy access and targeted drug delivery therapy by avoidance of the straight line path of normal linear needles [3]. The increasing complexity invariable leads to increased cost of the devices and there is a need to balance these conflicting aims.

We report our preliminary experience with a CT or PETCT-guided robotic positioning system (ROBIO ${ }^{\text {TM }}$ EX, Perfint Healthcare Pvt. Ltd, Florence, OR, USA), which is designed to assist interventional radiologists in performing procedures that require precise tool positioning. ROBIO ${ }^{\mathrm{TM}} \mathrm{EX}$ is a standalone positioning device that can be moved to the desired position along the patient table of the CT system. This device has two linear motions to position the guide to the point of interest and two angular motions to facilitate the angular entry of the needle. The system offers several features to help clinicians to target the tumour and plan for accurate tool placement. It facilitates targeting and tool placement in deepseated lesions requiring orbital, cranio-caudal angulations or a combination of both for thoracic, abdominal and pelvic interventional procedures.

\section{Materials and methods}

This study was granted with medical ethics approval (MEC no. 949.9) from the Medical Ethics Committee, University of Malaya Medical Centre, Kuala Lumpur, Malaysia.

\section{Patients}

A total of 11 patients with 17 lesions were treated with radiofrequency ablation (RFA) with the guidance of the ROBIO $^{\mathrm{TM}}$ EX (Perfint Healthcare Pvt. Ltd, Florence, OR, USA). Six patients had new and recurrent hepatocellular carcinoma (HCC) and 5 patients had colorectal metastases. Eight patients were treated with the Cool-tip RFA system (Valleylab, Boulder, CO, USA) and 3 patients were treated with the RITA StarBurst ${ }^{\circledR}$ system (Angiodynamics, Latham, NY, USA). All the lesions were no greater than $3.0 \mathrm{~cm}$ in maximum diameter (the average dimension of the tumour was $2.0 \times 2.2 \mathrm{~cm})$.

All the RFA procedures were performed under general anaesthesia. Once the patients were intubated, they were wrapped in reusable immobiliser to minimise patient movement during the procedure. In order to optimise needle placements, the baseline $\mathrm{CT}$, verification of needle placement and post-procedure $\mathrm{CT}$ were performed at end expiration with the airway disconnected from the ventilator. Additionally, to minimise liver excursion between the end expiration (when needle placement was carried out) and the inspiration, the tidal volumes were set at low with high respiratory rate and high $\mathrm{O}_{2}$ level. Further, to ensure that spontaneous breathing of the patient would not affect the end expiratory phase, we used muscle relaxants regularly especially when doing multiple placements. Otherwise the loss of muscle paralysis would impair the end tidal volume and place the liver at a much lower level.

All the patients had non-contrast baseline $\mathrm{CT}$ to identify the lesions. However in 6 patients, because the lesions were small, contrast-enhanced CT studies (example shown in Fig. 1a) were performed as the baseline to better delineate the location of the lesions. Post-RFA three-phase CTs (Fig. 1d) were then performed to assess the completion of the ablation as well as to act as the baseline for subsequent follow-up. One patient, however, did not have post-RFA CT because of renal impairment.

\section{ROBIO $^{\text {TM }}$ EX treatment planning}

Following baseline CT, the lesions were identified. The CT images were exported to the ROBIO ${ }^{\mathrm{TM}} \mathrm{EX}$ workstation for treatment planning. The target point (centre of the tumour volume) as well as the entry point (needle puncture site on skin surface) was determined by the interventional radiologist. The angulations of the needle, the depth of the lesion as well as the needle trajectory path were calculated by the ROBIOTM EX workstation and shown on the treatment plan (Fig. 1b). The plan was carefully checked by the radiologist to avoid critical organs or bone across the trajectory. Once confirmed, the plan was sent to the robotic arm for execution.

\section{Robotic-assisted needle placement}

Once the treatment plan was confirmed, the patient was positioned to the exact coordinates as shown in the ROBIO ${ }^{\mathrm{TM}} \mathrm{EX}$ treatment plan. The patient's skin was prepared for the procedure in the intended region. The robotic arm was then activated and it moved automatically to the planned coordinates as determined in the treatment plan. Once the robotic arm was completely halted at its position, the radiologist placed an appropriate bush and bush holder at the end-effector of the arm (Fig. 2). The skin and liver capsule along the projected path of the RFA needle were infiltrated with $10 \mathrm{~mL}$ of $1 \%$ lignocaine. The radiologist then inserted the RFA needle through the bush and pushed the needle to the predetermined depth where the end-effector was located (Fig. 3). Upon completion of the insertion of the RFA needle, the robotic arm was detached from the needle and returned to its original position. CT fluoroscopy (Fig. 1c) was performed to ensure that the RFA needle was located within the tumour volume. RFA therapy was then started and the completeness of the ablation was determined by using multiphasic contrastenhanced CT immediately after the RFA. 
Data collection and analysis

The orbital and cranio-caudal angulations of the robotic arm were recorded for each lesion targeted in all patients. The number of adjustments of the RFA needle was documented. Deviation of the tip from the centre of the targeted location was recorded.

The performance level of the overall procedures was assessed by the interventional radiologist for each robotic-assisted RFA on a five-point scale $(5=$ excellent, $4=$ good, $3=$ average, $2=$ fair and $1=$ poor). Any complications related to the use of the robot or the RFA were also recorded.

The CT fluoroscopic dose (DLP) received by the patients during the needle placement and ablation was recorded. The total dose from the whole procedure including the multiphasic $\mathrm{CT}$ studies was also recorded as the $\mathrm{CTDI}_{\mathrm{vol}}$. The doses were then compared with a random historical control group of patients who had liver RFA performed by the same radiologist but without using the assistance of a robot for needle placement.

\section{Results}

Radiofrequency ablation was successfully completed in 11 patients with 17 lesions. The deepest lesion was $13.7 \mathrm{~cm}$ and the shallowest was $6.2 \mathrm{~cm}$ from the skin surface. The diameter of the lesions ranged from 1.1 to $3.0 \mathrm{~cm}$. The lesions were all targeted successfully with the assistance of a robot. No repositioning of the needle was required in any of the patients. The orbital angulations of the robotic arm ranged from $-49.0^{\circ}$ to $46.5^{\circ}$ (mean negative angulation was $-26.5 \pm 24.9^{\circ}$; mean positive angulation was $\left.27.3 \pm 12.0^{\circ}\right)$. The cranio-caudal angulations remained at $0^{\circ}$ in 9 lesions ( 6 patients) whereas the remaining 8 lesions (3 patients) had cranio-caudal angulations of up to $25.0^{\circ}$ (mean $7.4 \pm 9.9^{\circ}$ ).

Readjustments of the RFA needle were necessary in 6 lesions, with single readjustment in 4 lesions and two readjustments in the remaining 2 lesions.

The total DLP and CTDI $\mathrm{v}_{\mathrm{vol}}$ dose for the entire procedure were $956.09 \pm 400.33 \mathrm{mGy} \mathrm{cm}$ and $258.00 \pm 125.46 \mathrm{mGy}$, respectively. Compared with historical data from our standard RFA procedure $(n=30)$, the total DLP and CTDI ${ }_{\mathrm{vol}}$ dose were $1,703.93 \pm 1,152.37 \mathrm{mGy} \mathrm{cm}$ and $632.73 \pm 503.06 \mathrm{mGy}$, respectively.

All patients had successful ablation confirmed on multiphasic contrast-enhanced CT except in one patient who had renal impairment, which precluded contrast injections.

Post-RFA contrast-enhanced CT was performed in all except one patient owing to renal impairment. The CT images showed successful ablation of the targeted lesions in all patients. No complications related to either the robot or the RFA were noted in this study.
The mean performance level rated for the robotic-assisted RFA procedure was $4.6 \pm 0.5$, in which the score 5 was achieved in 7 patients and the score 4 was achieved in the remaining 4 patients. The patients' demography, treatment protocols, radiation dose and evaluation of treatment outcomes are summarized in Table 1.

\section{Discussion}

Image guidance techniques have revolutionised the performance of interventions in medicine developed from the use of advanced imaging investigations. These developments have been adapted for neurosurgery, orthopaedic procedures, urological surgery, etc. Current research into the combined application of image-guided surgery and robots with the complexities of soft tissue registration, operative navigation and surgical use presents unique engineering challenges and new knowledge requirements for interventional radiology.

Recent advances in robotically guided interventions have been successful in assisting placement of needles or related instruments for surgery or interventional procedures [4-9]. Magnetic resonance imaging (MRI)-compatible robots have also been developed despite their significant engineering challenges and are continuing to be investigated for prostate biopsy utilising the potential advantages of multiparametric MRI. There may also be a future role for improving the accuracy and precision of radioactive seed placement for prostate cancer using the interventional robotic device [10].

The robot used in this study was a CT- or PET-CT-guided needle positioning system for interventional procedures. The system calculates coordinates on DICOM images from CT or PET-CT and guides the placement of the needle accurately within the body using a robotic arm. The depth of needle placement is pre-determined by the system but the operator still has the option of varying this for increased safety. The system can be used for tumour targeting for abdominal and thoracic interventions, including biopsy, fine needle aspiration cytology (FNAC), pain management, drainage and tumour ablation.

Earlier robotic guidance devices required extensive installation and were often cumbersome and occupied a lot of space in the operation room $[6,11,12]$. Devices that are time consuming in terms of pre-arrangement and usage are economically unattractive and are therefore not likely to be used in daily routine. $\mathrm{ROBIO}{ }^{\mathrm{TM}} \mathrm{EX}$ requires minimal effort to be mounted and registered to the CT device using the InstaRegTM technology (Perfint Healthcare Pvt Ltd., Florence, OR, USA). The system is motorised and can be operated by one person. These features reduced the complexity of the robotic-guided procedure.

Localisation and navigation of the robots are usually performed with optical or magnetic localisation spheres, 
Fig. 1 a Contrast-enhanced baseline CT image shows solitary colorectal metastases $(26.2 \mathrm{~mm}$ diameter) in segment VI. b Reconstructed CT images (slice thickness $1 \mathrm{~mm}$ ) were sent to the ROBIO $^{\text {TM }}$ EX workstation for treatment planning. The simulated needle trajectory path was shown on the treatment plan and verified by the radiologist. c A CT fluoroscopy check was carried out to verify the accuracy of the needle placement within the target volume. d Post-RFA threephase CTs to assess the completeness of tumour ablation a

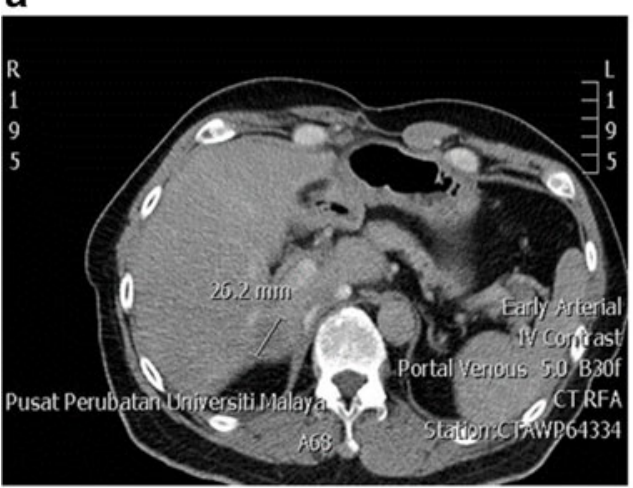

c

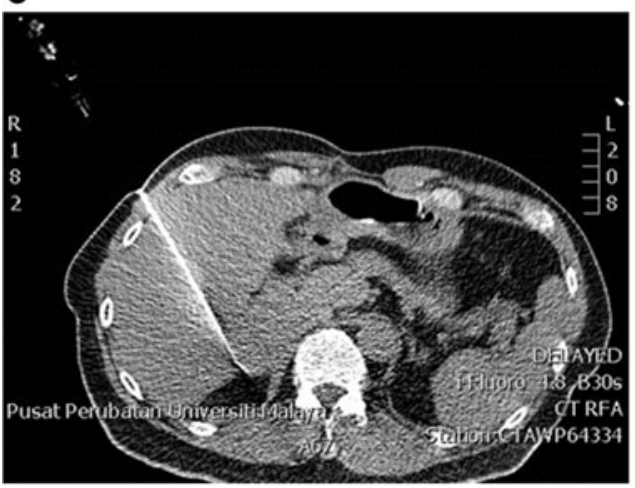

b

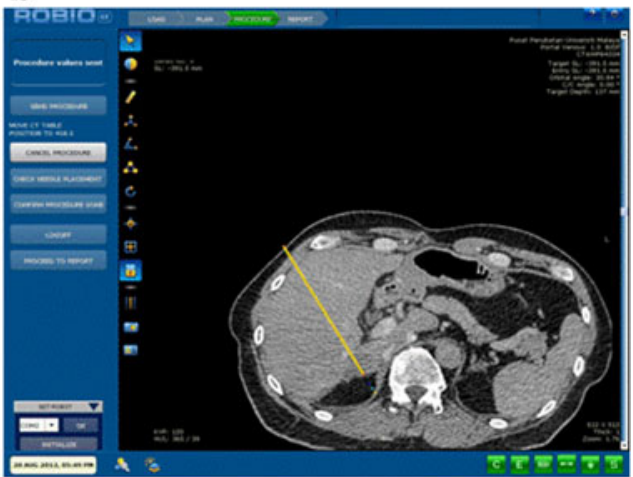

d

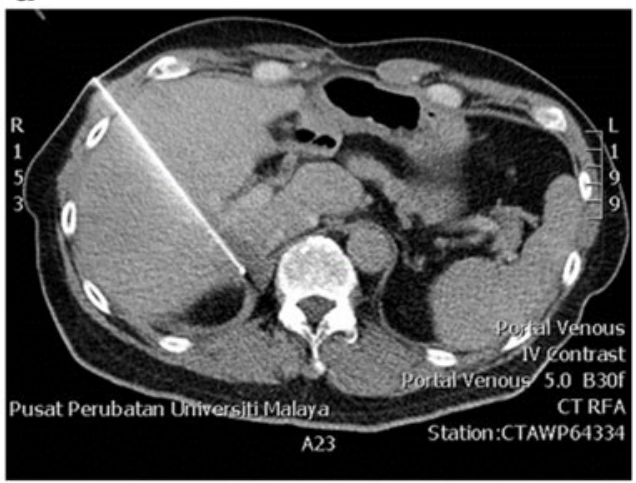

requiring a pre-procedure import and processing of the $3 \mathrm{D}$ data to the robot's workstation, which can be a complex and time-consuming procedure. However in our preliminary experience with the Robio ${ }^{\mathrm{TM}} \mathrm{EX}$, we found the overall satisfaction with the performance to be high. Even though the planning did take time, it was found to be intuitive and this

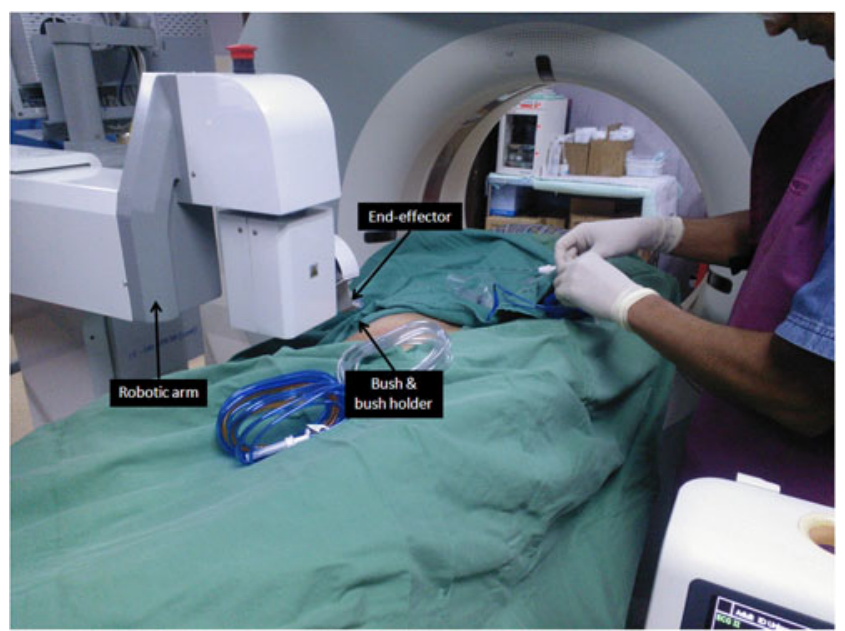

Fig. 2 The robotic arm was positioned automatically to the exact coordinates according to the treatment plan. The bush and bush holder were clamped firmly at the end-effector of the robotic arm before insertion of the RFA needle through the bush increased time was compensated for by greater speed and accuracy in placing the RFA needles.

Most importantly, the greater control and ease of needle placement outside the bore of the CT gantry without exposure to ionising radiation was a tremendous benefit. Both before and during the needle insertion, neither direct exposure of the interventionist's hands to the radiation beam was involved, nor was the use of inaccurate holding devices such as forceps necessary. During the conventional CT-guided RFA procedure, insertion and placement of the RFA needle are performed manually under the guidance of real-time CT fluoroscopy. This challenging procedure needs to be performed by an experienced interventional radiologist and certain impreciseness during the manual insertion is unavoidable. The continuous reassessment and repetitive corrections of the needle orientation under the guidance of CT fluoroscopy could lead to an increase in radiation exposure to the patients as well as the attending staff. Our study showed a significant reduction of CT fluoroscopic dose in patients of $43.9 \%$ (DLP) and $59.2 \%\left(\mathrm{CTDI}_{\mathrm{vol}}\right)$ comparing robotic and non-robotic-assisted RFA for HCC. This comparison might be biased because the radiation dosimetry data for conventional RFA were collected from our historical HCC patients in our hospital database. Further the interventional radiologist who participated in this study was aware of the objective of dose assessment; therefore, there might be unintended biasness in reducing the fluoroscopic dose. A randomised controlled study with a larger sample size would be necessary to confirm this. 


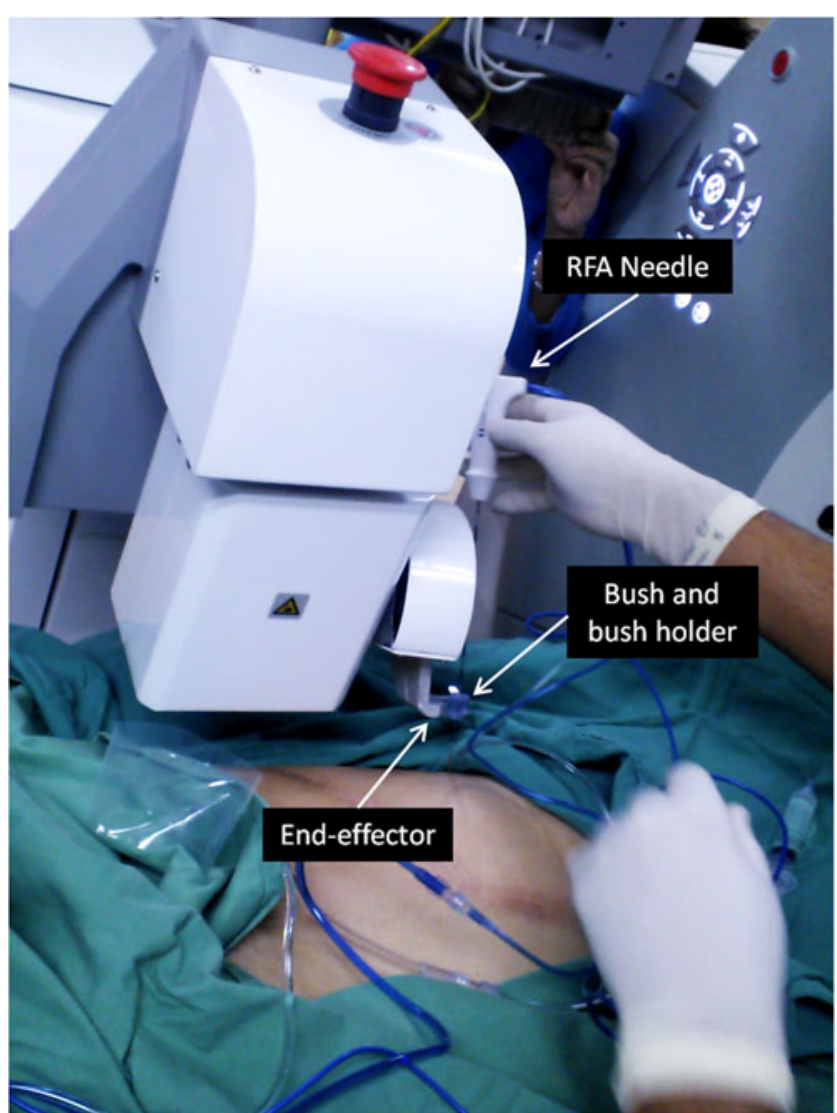

Fig. 3 The RFA needle was inserted by the radiologist through the bush and the bush holder. The needle was then pushed to the predetermined depth where the end-effector of the robotic arm was located. The robotic arm was then detached from the RFA needle to allow a CT check of positioning

Although we did not specifically measure the set-up time in the patients treated, the interval between docking the device until it was finally attached and powered up was less than $5 \mathrm{~min}$. The time until the acquisition and planning were completed took an average of another $10 \mathrm{~min}$. Although there is an initial set-up time for the robot and planning, this can be compensated for by reduced need (or time) of needle repositioning using the manual method. Future analysis is proposed to evaluate the time efficiency of the whole procedure.

We worked closely with anaesthetists in this study to optimise needle placements. It was noted that performing all procedures at the end of expiration with the airway disconnected from ventilator produced consistent positing. Additionally using low tidal volumes with high respiratory rate and high $\mathrm{O}_{2}$ was useful to minimise liver excursion and needle movement in the craniocaudal direction. Further to ensure that spontaneous breathing of the patient would not affect the end expiratory phase, we ensured that muscle relaxants were used regularly especially when doing multiple placements. Otherwise the loss of muscle paralysis would impair the end tidal volume and place the liver at a much lower level. The baseline CT, needle placement and post-procedure CT acquisitions were all performed at the end of expiration once the ventilator was disabled. Others have suggested that anaesthetic manoeuvres, such as high frequency jet ventilation, to reduce respiratory motion significantly reduce radiation dose [13]. However these systems are expensive and require a greater skill set.

There was no multiplanar capability of the current ROBIO ${ }^{\mathrm{TM}}$ EX system and thus determining the centre of the lesion using axial imaging may be limited. Also if there is more than a single lesion, the operator needs to plan the subsequent treatments one at time. Besides, the guidance software is also yet to compensate for movements of the target region, especially those caused by respiration as the planned trajectory is based on a static-acquired 3D data set. As the procedure was performed on patients under arrested end expiration we were able to achieve more consistent locations of the target lesions and hence accurate deployment of the needles and measuring of the outcomes. The use of the breath-holding systems to "fix" the location of the lesions to a pre-determined point requires that the patients fully understand and are able to cooperate completely with the requirements. This would also add to the time required for training of the patients before the actual procedure.

In addition, the use of robotic-assisted RFA may require a change to the current workflow. Although it adds more steps to the procedure (mainly done by the technician for device setup) it does not bring any significant change in the clinical workflow of the clinician. With free-hand CT-guided procedures, once the studies have been viewed, the patient position can be determined. The radiographer would have done the baseline CT and the physician would decide the best approach. Once that was done the physician would localise the entry point using a laser and under fluoroscopic guidance the procedure would be over in less than $10 \mathrm{~min}$ in most circumstances. With the robot there are several additional steps, which include docking the robotic system, importing the images into the workstation, planning the entry and target points, inputting the length of the needle, and finally sending the information to the robotic arm. The robotic arm would then move automatically to the accurate target position for needle insertion. As a result, there would be a need to redefine the roles of different members of the medical team and the work flow chart. Also, the staff needs to being well versed with the robot and its operation.

In conclusion, we present our early experience of roboticassisted CT-guided RFA for both primary and secondary liver tumours. We have been able to show that the automated system works well and could provide technical and diagnostic success rates similar to those obtained with the manual method. Also, we found that the automated device decreased the number of needle position adjustments and thereby minimised the procedure time. The robotic device showed good accuracy for percutaneous needle placement for RFA therapy with a lower radiation dose compared with historical controls. From our preliminary study we found that the robot provides high accuracy with only a few readjustments required. Even though these preliminary data were promising, the study was not 


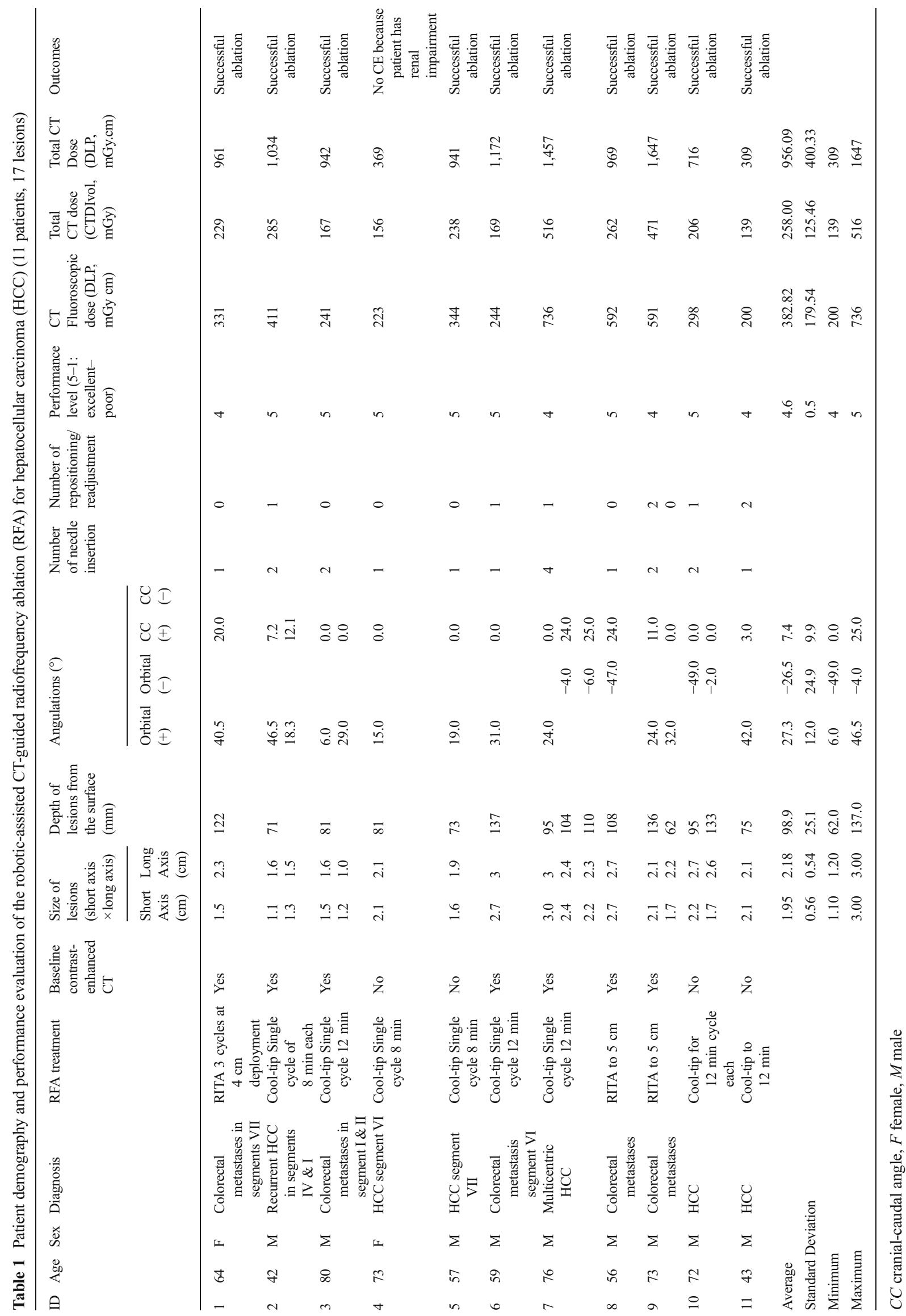


randomised. In future a randomised study comparing the robotic and non-robotic-assisted RFA needs to be carried out with a larger sample size to determine the cost-effectiveness in terms of time, cost and radiation dose to both patients and operators. There is also a need for larger multi-centre studies for cross-centre comparison.

Acknowledgments This study was supported in part by Perfint Healthcare Pvt. Ltd, Florence, OR, USA which provided research funding and equipment. The authors had no pertinent conflict of interest and had unrestricted control of study data.

Open Access This article is distributed under the terms of the Creative Commons Attribution Noncommercial License which permits any noncommercial use, distribution, and reproduction in any medium, provided the original author(s) and the source are credited.

\section{References}

1. Mozer P, Troccaz J, Stoianovici D (2009) Urologic robots and future directions. Curr Opin Urol 19:114-119

2. Pollock R, Mozer P, Guzzo TJ et al (2010) Prospects in percutaneous ablative targeting: comparison of a computer-assisted navigation system and the AcuBot Robotic System. J Endourol 24:1269-1272

3. Rucker DC, Jones BA, Webster RJ 3rd (2010) A geometrically exact model for externally loaded concentric-tube continuum robots. IEEE Trans Robot 26:769-780
4. Cleary K, Melzer A, Watson V, Kronreif G, Stoianovici D (2006) Interventional robotic systems: applications and technology state-ofthe-art. Minim Invasive Ther Allied Technol 15:101-113

5. Fichtinger G, Fiene JP, Kennedy CW et al (2008) Robotic assistance for ultrasound-guided prostate brachytherapy. Med Image Anal 12:535-545

6. Onogi S, Morimoto K, Sakuma I et al (2005) Development of the needle insertion robot for percutaneous vertebroplasty. Med Image Comput Comput Assist Interv 8:105-113

7. Penzkofer T, Isfort P, Bruners $P$ et al (2010) Robot arm based flat panel CT-guided electromagnetic tracked spine interventions: phantom and animal model experiments. Eur Radiol 20:2656-2662

8. Rasmus M, Huegli RW, Bilecen D, Jacob AL (2007) Robotically assisted CT-based procedures. Minim Invasive Ther Allied Technol 16:212-216

9. Zangos S, Melzer A, Eichler K et al (2011) MR-compatible assistance system for biopsy in a high-field-strength system: initial results in patients with suspicious prostate lesions. Radiology 259:903-910

10. Bonekamp D, Jacobs MA, El-Khouli R, Stoianovici D, Macura KJ (2011) Advancements in MR imaging of the prostate: from diagnosis to interventions. Radiographics 31:677-703

11. Tovar-Arriaga S, Tita R, Pedraza-Ortega JC, Gorrostieta E, Kalender WA (2011) Development of a robotic FD-CT-guided navigation system for needle placement-preliminary accuracy tests. Int J Med Robot 7:225-236

12. Yanof J, Haaga J, Klahr P et al (2001) CT-integrated robot for interventional procedures: preliminary experiment and computerhuman interfaces. Comput Aided Surg 6:352-359

13. Abderhalden S, Biro P, Hechelhammer L, Pfiffner R, Pfammatter T (2011) CT-guided navigation of percutaneous hepatic and renal radiofrequency ablation under high-frequency jet ventilation: feasibility study. J Vasc Interv Radiol 22:1275-1278 